\title{
Distributed Monitoring and Control of Future Power Systems via Grid Computing
}

\author{
G. A. Taylor, M. R. Irving Member, IEEE, P. R. Hobson, C. Huang, P. Kyberd and R. J. Taylor
}

\begin{abstract}
It is now widely accepted within the electrical power supply industry that future power systems will operate with significantly larger numbers of small-scale highly dispersed generation units that use renewable energy sources and also reduce carbon dioxide emissions. In order to operate such future power systems securely and efficiently it will be necessary to monitor and control output levels and scheduling when connecting such generation to a power system especially when it is typically embedded at the distribution level. Traditional monitoring and control technology that is currently employed at the transmission level is highly centralized and not scalable to include such significant increases in distributed and embedded generation. However, this paper proposes and demonstrates the adoption of a relatively new technology 'Grid Computing' that can provide both a scalable and universally adoptable solution to the problems associated with the distributed monitoring and control of future power systems.
\end{abstract}

Index Terms-- Distributed Energy Resources, Distributed Monitoring and Control, Grid Computing, Renewable Energy Sources.

\section{INTRODUCTION}

$\mathrm{S}_{\mathrm{p}}^{\mathrm{i}}$ gnificantly large-scale increases in sustainable electrical power generation are major global developments that will affect the design and operation of future power systems [1]. Another important consideration is the ongoing global restructuring and deregulation of electricity industries [2], which has lead to the emergence of competitive market players and a move away from vertically integrated organizational structures to more disparate structures [2].

The research described in this paper addresses these major issues and seeks to provide a novel and universally adoptable grid computing approach [3,4]. Essentially this approach would develop and extend Grid Computing techniques that are currently being applied by the e-science community in order to solve massively large-scale computational problems in engineering and science $[3,4]$. Such problems currently require the processing, analysis and archival of petabytes of data via large-scale coordination of vast computational resources that are widely distributed across the Internet [3,4].

This work was supported in part by the EU under the GRIDCC Grant.

G. A. Taylor and M. R. Irving, Brunel Institute of Power Systems, Brunel University, Uxbridge, Middlesex, UB8 3PH, UK (e-mail: Gareth.Taylor@brunel.ac.uk).

P. R. Hobson, C. Huang, P. Kyberd and R. J. Taylor, Sensors \& Instrumentation Research Group, Brunel University, Uxbridge, Middlesex, UB8 3PH, UK (e-mail: Peter.Hobson@brunel.ac.uk).
Traditional monitoring and control of power systems is highly centralized and occurs over dedicated and private Wide Area Networks (WANs) [5]. Recently, considerable research effort has been applied to the monitoring and control of power systems using parallel and distributed processing across such networks $[5,6,7,8]$. However, the majority of the research has utilized existing network protocols $[7,8]$ and telecommunication standards to develop non-standardized distributed platforms on specific communication networks $[5,6]$. Such approaches can provide immediate benefits on existing infrastructure [5], but do not easily provide an extendable or adoptable infrastructure and limit flexibility with regard to developing and extending future highly distributed monitoring and control systems [5]. Similarly, considerable research is underway with regard to employing distributed monitoring and control in micro-grids in future power systems with increased amounts of Distributed Energy Resources (DER) [9,10,11]. However, further research is required with regard to developing a flexible, extendable and universally adoptable platform [12]. Such a platform must facilitate the coordination of future heterogeneous micro-grids and other disparate power system and network entities that are essentially independent, but are technically required to cooperate within a virtual organization in order to effectively monitor and control increasingly complex power systems and networks [12]. Key issues associated with future monitoring and control of power systems with increased distributed amounts of embedded generation require addressing.

The major areas of research that need to be further addressed when considering the development of universal standards for distributed monitoring and control of power systems can be categorized as follows [2];

- Algorithmic procedures for performing parallel and distributed processing $[5,6,7,8]$.

- Standards for information and data exchange $[5,13,14]$.

- Middleware for the processing and exchange of data between distributed processes and agents $[5,9,10,11]$.

Grid Computing is being specifically researched and developed by the e-science community in order to universally facilitate the latter two areas and provide an open standard 'plug in' approach to the first area of research [3,4]. A combination of public and private communication networks can be inherently employed with regard to Grid Computing as it is being developed as an extension of the existing Internet. The development of Grid Computing is analogous with the early development of the Web. Levels of data security and 
Quality of Service (QoS) can be guaranteed through service level agreements between Grid Computing entities and Internet Service Providers [3,4].

Initially the UK e-science community has applied Grid Computing to off-line analyses and simulations of engineering and science problems [15]. Therefore, currently established Grid Computing middleware such as the Globus Toolkit [16] has been developed to offer a platform that facilitates a batch mode execution and scheduling of processes. Current research is being undertaken by the authors of this paper to design and develop enhanced middleware that is applicable to the on-line distributed monitoring and control of power systems and networks [17,12].

The remainder of this paper is organized as follows; Section II provides an overview of the proposed power system Grid Computing platform; Section III presents two cases that demonstrate the application of the power system Grid Computing platform; Section IV concludes the paper and describes further research

\section{OVERVIEW OF GRID COMPUTING PLATFORM}

In the following section it is assumed that in all generators are equipped with computational and communication facilities. Such an assumption is congruent with the currently increasing number of small and large-scale devices being connected to the Internet.

\section{A. Distributed Monitoring and Control}

It is now widely accepted that a large numbers of smaller generators will be introduced in the coming decades, utilising renewable energy and reducing carbon emissions. As these generators are connected into the power system (often embedded at distribution level) it will become necessary to monitor and control their output level and their on/off schedule. The standard control technology in use at transmission level is not scalable to very large numbers of generators. Grid Computing can provide a relatively inexpensive new technology, allowing the output of embedded generators to be monitored and when necessary controlled. An outline of the power systems Grid-computing platform that has been adopted for the research presented in this paper is illustrated in Figure 1.

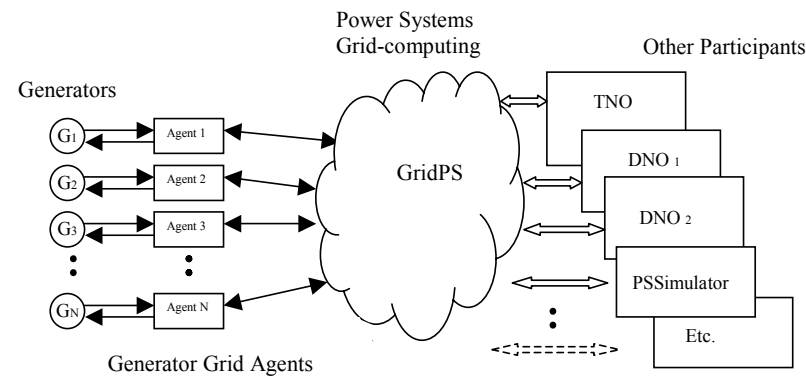

Fig. 1. Overview of a power systems Grid Computing platform.

\section{DEMONSTRATION OF GRID COMPUTING PLATFORM}

\section{A. Distributed Real Time Power System Simulator}

The Brunel Institute of Power Systems is collaborating with the Sensors \& Instrumentation Research Group of Brunel University as part of the contribution to the GRIDCC project [17]. The collaboration involves performing realistic computer simulation of electrical transmission networks in order to demonstrate the Grid Computing platform that is being developed within the GRIDCC project [17].

One of the goals of the GRIDCC project was to demonstrate that an existing, monolithic legacy software package, the Power System Simulator (PSSimulator) [20] that was written in FORTRAN 77 and compiled on MS Windows platforms could be executed on a modern Grid computing platform. The FORTRAN driver routine was replaced with a $\mathrm{C}++$ wrapper for the library. This facilitates the communication with the Grid Computing middleware, and in the meantime expands the $\mathrm{I} / \mathrm{O}$ possibilities beyond the text file $\mathrm{I} / \mathrm{O}$ used in the original version of the program [20]. Enhanced performance is provided through the employment of threads and a schematic of the deployment of the software on a Grid Computing platform is illustrated in Figure 2. However, it should be noted that the power network demonstrated in this paper is a very simple case that presents no issues with regard to executing the simulation in real time on a single processor. The objective of porting the application to a Grid Computing platform has been achieved, with the PSSimulator now running under the gcc compiler on machines using the Scientific Linux 3 operating system. In the following section we demonstrate the distributed application of the PSSimulator with the use of the Grid Computing middleware candidate Narada Brokering [18] in order to implement a light-weight distributed monitoring facility.

As a further goal of the GRIDCC project [17] the initial middleware candidate will be replaced by the Instrument Element middleware that is currently being developed as part of the GRIDCC project [17].

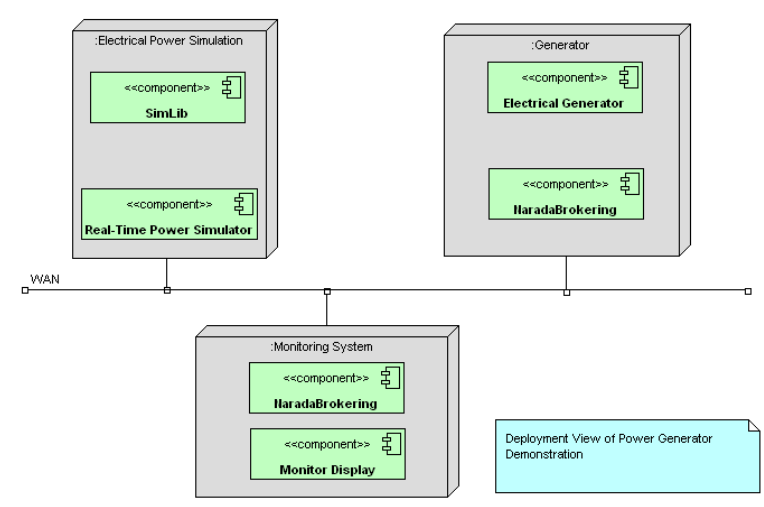

Fig. 2. A UML deployment view of the Grid Computing nodes and their components. 
The PSSimulator is a sophisticated software application [20] that simulates the realistic operational modes of conventional large electricity generators plus a network model of transmission lines, transformers and other components. Generators can be in a number of states; Not Generating; Synchronising; Running etc. and their delivered power and $\mathrm{AC}$ frequency will vary with time.

Figure 1 illustrates an initial deployment of the software in order to demonstrate the Grid Computing platform. In this deployment the instances of the electrical generators have been executed in a distributed fashion on machines in Italy, Switzerland and the UK. At present the instances only act as message forwarding agents for their representations inside the PSSimulator package that is executed on a local machine. The generator instance then re-publishes the information using NaradaBrokering and the messages are then received and displayed by a light-weight monitoring client.

\section{B. Middleware - Narada Brokering}

The distributed application of the PSSimulator uses Narada Brokering [18] which is asynchronous message oriented middleware that employs a publish and subscribe model. A variety of Internet transport and application layer protocols are supported, that includes TCP, UDP and HTTP. The middleware also supports robust messaging and several Web Service specifications such as WS-Eventing and WSReliableMessaging [19].

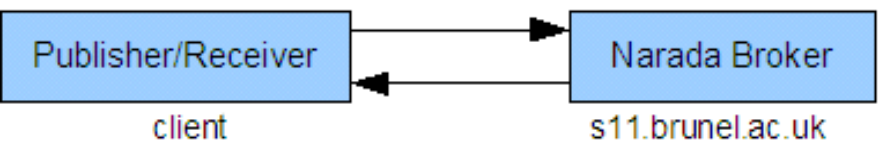

Fig. 3. Computer configuration for demonstration of Narada Brokering.

TABLE I

HARDWARE, PlATFORM AND SOFTWARE SPECIFICATION FOR DEMONSTRATION

\begin{tabular}{|c|l|l|l|l|}
\hline \multicolumn{1}{|c|}{ Computer } & \multicolumn{1}{c|}{$\begin{array}{c}\text { CPU/ } \\
\text { Memory }\end{array}$} & \multicolumn{1}{c|}{ OS } & \multicolumn{1}{c|}{ Java } & \multicolumn{1}{c|}{ Software } \\
\hline s11.brunel.ac.uk & $\begin{array}{l}\text { Pentium 4 } \\
3 \mathrm{GHz}, \\
1 \mathrm{~GB}\end{array}$ & $\begin{array}{l}\text { Scientific } \\
\text { Linux, kernel }\end{array}$ & $\begin{array}{l}\text { J2se jdk } \\
1.4 .2\end{array}$ & $\begin{array}{l}\text { Narada } \\
\text { Brokering }\end{array}$ \\
\hline client & $\begin{array}{l}\text { Celeron } \\
1.3 \mathrm{GHz}, \\
512 \mathrm{MB}\end{array}$ & $\begin{array}{l}\text { Scientific } \\
\text { Linux, kernel }\end{array}$ & $\begin{array}{l}\text { J2se jre } \\
1.4 .2\end{array}$ & PS Simulator \\
\hline
\end{tabular}

The purpose of this demonstration is to measure the round trip time of Narada Brokering and check that the results satisfy the real-time requirement of the Power Grid. In this demonstration we have developed a Java program to simulate the operational activities of the power generators as illustrated in Figure 1. We have used the program to measure the realtime performance of Narada Brokering. The Java program forks into up to 1000 threads, where each thread simulates one power generator. The threads start one by one in intervals of 1 second and last 20 minutes. It takes approximately 17 minutes for all the threads to start up. Each thread sends one large message at startup (1 KB) and then sends one small message to Narada Brokering every ten seconds. Another receiver program subscribes to Narada Brokering and receives all the messages [18]. The two programs are located on one machine and the round trip time is calculated at the end of the test. In this demonstration we have adopted TCP as the underlying transport layer connection protocol. However, in future demonstrations the connectionless transport layer protocol UDP will be employed in order to improve speed and efficiency.

\section{Initial Results}

The horizontal axis in Figure 4 is the number of messages and the vertical axis is the round trip time of the messages. As we can see from the graph, the majority of the Round Trip Time delays are within 0.1 second and all of them are within 0.3 second.

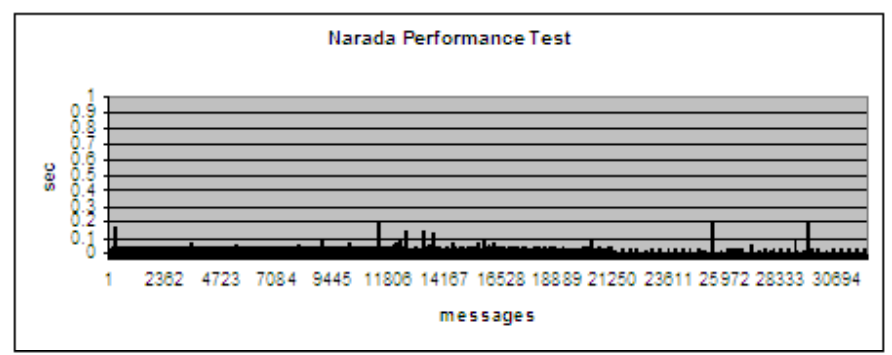

Fig. 4. Round Trip Times obtained from the Narada Brokering demonstration.

Figure 5 illustrates part of the NetLogger visualization of the Narada Brokering demonstration. Each red line represents the lifeline of one message. The vertical axis shows the events, before_sending and received. The horizontal axis is the timescale and each division is 0.1 second. As we can see from the graph, there are up to ten messages sent within 0.1 second and they all arrived within 0.1 second.

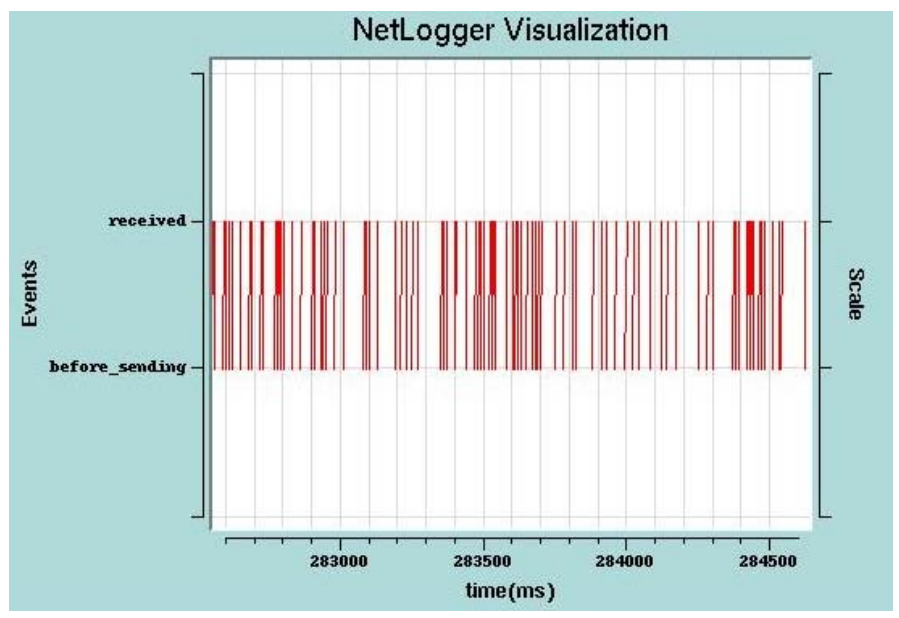

Fig. 4. NetLogger visualization of the Narada Brokering demonstration.

\section{CONCLUSIONS AND FURTHER RESEARCH}

The test results indicate that the performance of Narada Brokering middleware fully satisfies the real-time requirement of the Power Grid, which in this research has been selected as 5 seconds. Such a value is realistic value as there will be a 
limit on the total amount of data that can be monitored and processed by any decentralized control scheme. NaradaBrokering has a better performance than R-GMA. Therefore at this stage of the research we can conclude that Narada Brokering has excellent performance and that it satisfies the initial requirements of a Grid Computing Platform. It is also important to note that the Grid Computing platform as described in Figure 1 can be extended in a generic fashion to include a wide range of RES facilities such as Inverters, $\mathrm{CHP}, \mathrm{PV}$, wind turbine etc.

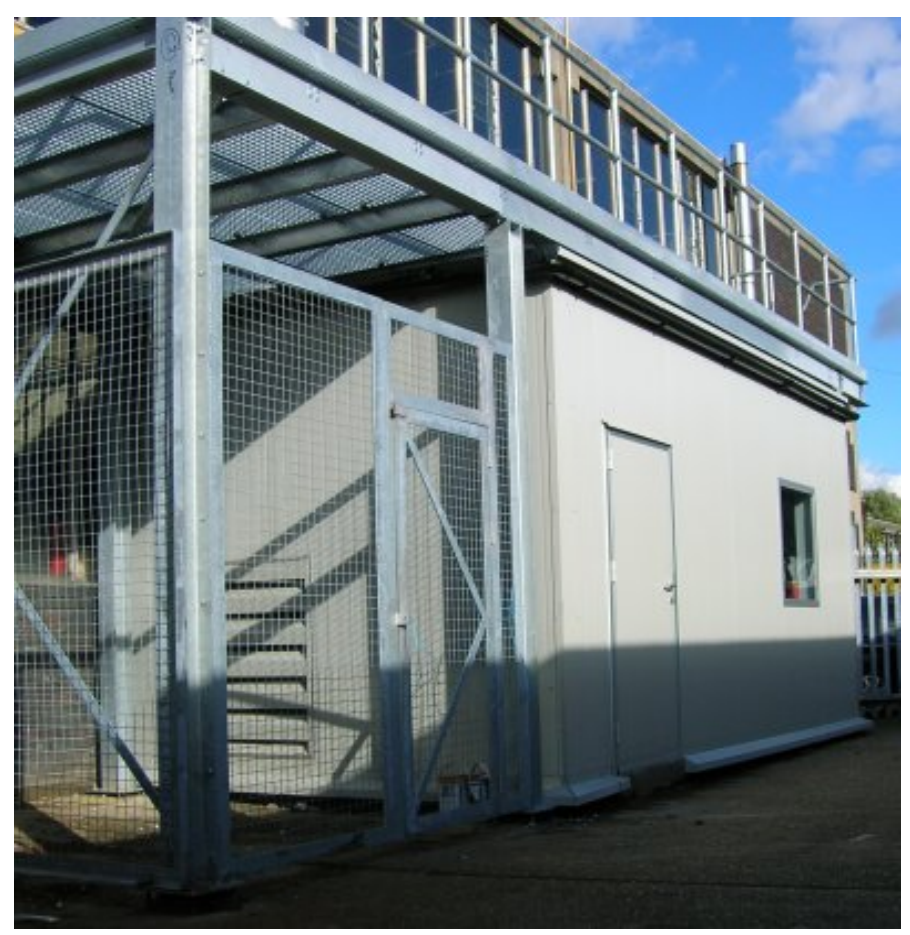

Fig. 5. Newly installed (October 2005) Combined Heat \& Power and PV experimental facility.

The main objectives of the ongoing research can be summarized as follows;

- Completely decouple the generator simulation from the electrical network simulation. Enabling realistic simulation of large numbers of geographically distributed generators.

- Replace the light-weight monitor with the full IE package developed as part of the GRIDCC project.

- Implement and demonstrate the monitoring and messaging middleware.

- Implement appropriate levels of security within the middleware.

In addition, further experimental developments at Brunel University will give us monitoring access to real power generator instruments, for example an $80 \mathrm{~kW}$ Combined Heat \& Power Unit as illustrated in Figure 5 that includes photovoltaic array on the roof of the structure and a planned $(1 \mathrm{~kW})$ wind turbine. As part of the research project we will have access to the real-time electrical power data produced by this system.

\section{REFERENCES}

[1] G.M. Masters, Renewable and Efficient Electric Power Systems, John Wiley \& Sons, 2004, Ch. 4.

[2] G.M. Masters, Renewable and Efficient Electric Power Systems, John Wiley \& Sons, 2004, Ch. 3.

[3] I. Foster and C. Kesselman (eds.): The Grid: Blueprint for a New Computing Infrastructure, Morgan Kaufmann, 1999.

[4] F. Berman, G. Fox and A.J.G. Hey, Grid Computing: Making the Global Infrastructure a Reality, John Wiley \& Sons, 2003.

[5] M. Shahidehpour and Y. Wang, Communication and Control in Electric Power Systems; Appications of parallel and distributed processing, IEEE Press, 2003.

[6] J. Contreras, A. Losi, M. Russo and F.F. Wu, 'Simulation and Evaluation of Optimization Problem Solutions in Distributed Energy Management Systems', IEEE Trans. on Power Systems. 2002, Vol. 17, no. 1, pgs. 57-62.

[7] P.N. Biskas, A.G. Bakirtzis, N.I. Macheras, N.K. Pasialis, 'A decentralized implementation of DC optimal power flow on a network of computers', IEEE Trans. on Power Systems. 2005, Vol. 20, no. 1, pgs. 25-33.

[8] J. Shu, W. Xue, W. Zheng, 'A parallel transient stability simulation for power systems', IEEE Trans. on Power Systems. 2005, Vol. 20, no. 4, pgs. $1683-1689$.

[9] A.L. Dimeas, N.D. Hatziargyriou, 'Operation of a Multiagent System for Microgrid Control', IEEE Trans. on Power Systems. 2005, Vol. 20, no. 3, pgs. 1447- 1455 .

[10] M.M. Nordman, M. Lehtonen, 'Distributed agent-based State estimation for electrical distribution networks', IEEE Trans. on Power Systems. 2005, Vol. 20, no. 2, pgs. 652- 658.

[11] J.A. Hossack, J Menal, S.D.J. McArthur, J. R. McDonald, 'A multiagent architecture for protection engineering diagnostic assistance', IEEE Trans. on Power Systems. 2003, Vol. 18, no. 2, pgs. 652- 658.

[12] M.R. Irving, G.A. Taylor and P. Hobson, 'Plug in to Grid-Computing', IEEE Power and Energy Magazine. 2004, Vol. 2 No. 2, Pgs. 40-44.

[13] J.P. Britton, A.N. deVos, 'CIM-based standards and CIM evolution', IEEE Trans. on Power Systems. 2005, Vol. 20, no. 2, pgs. 758- 764.

[14] A.W. McMorran, G.W. Ault, I.M. Elders, C.E.T. Foote, G.M. Burt, J.R. McDonald, 'Translating CIM XML power system data to a proprietary format for system simulation', IEEE Trans. on Power Systems. 2004, Vol. 19, no. 1, pgs. 229- 235.

[15] T. Hey and A. Trefethen, 'The UK e-Science Core Programme and the Grid', Future Generation Computing Systems. 2002, Vol. 18, pgs. 1017 (also available at www.research-councils.ac.uk/escience)

[16] P. Marsh, 'Get on the Grid', IEE Computing and Control Engineering, February/March 2005, pgs. 41-45.

[17] GridCC EU ICT Project, 'Grid Enabled Remote Instrumentation with Distributed Control and Computation', www.gridec.org (last accessed December 2005).

[18] Narada Brokering, www.naradabrokering.org (last accessed December 2005)

[19] G. Fox, S. Pallickara, M. Pierce, H. Gadgil, 'Building Messaging Substrates for Web and Grid Applications', Philisophical Transactions of the Royal.Society: Mathematical, Physical and Engineering Sciences, August 15 2005, vol. 363, no. 1833, pgs. 1757-1773.

[20] M.Rafian, M.J.H.Sterling and M.R.Irving: 'Real Time Power System Simulation', Proc. IEE C, 1987, Vol, 134, pgs. 206-223. 


\section{BIOGRAPHIES}

G. A. Taylor received his BSc from the University of London, UK in 1987 and MSc and PhD from the University of Greenwich, UK in 1992 and 1997, respectively. He was the National Grid UK post-doctoral scholar at Brunel University, UK from $2000-2003$. He is currently a lecturer within the subject area of Electronic and Computer Engineering, Brunel University, UK. His research interests include power system and network monitoring, control and optimization.

M. R. Irving is a Professor and Electronic and Computer Engineering subject leader at Brunel University, London, U.K. His research interests include power system optimization and operation. He is also a director of the Brunel Institute of Power Systems, Brunel University, London, UK.

P. R. Hobson was born in Edinburgh, UK in 1959. He graduated from Edinburgh University in with a BSc in Physics in 1981 and was awarded his $\mathrm{PhD}$ from University College London in 1985 . He is currently a Professor within the subject area of Electronic and Computer Engineering, Brunel University, UK. His research interests include Particle Physics and Grid Computing.

C. Huang is a research fellow in the subject area of Electronic and Computer Engineering at Brunel University, UK. He is currently researching and developing Grid Computing middleware for real-time grid computing applications in engineering and science as part of the EU funded GRIDCC project at Brunel University.

P. Kyberd is a Lecturer in Particle Physics Informatics within the subject area of Electronic and Computer Engineering at Brunel University, UK. He is currently involved in Particle Physics and Grid Computing research.

R. J. Taylor is a research fellow in the subject area of Electronic and Computer Engineering at Brunel University, UK. He is currently researching and developing large-scale engineering and science problem solvers on Grid Computing platforms as part of the EU funded GRIDCC project at Brunel University. 